\section{The Adipokine Omentin in Late-stage Rheumatoid Arthritis and Endstage Osteoarthritis}

\section{To the Editor:}

In the past several years, adipokines have gained a lot of attention in the field of rheumatic diseases ${ }^{1}$. Rheumatoid arthritis (RA), for example, is associated with an altered production of adipokines ${ }^{1}$, and there is some evidence of involvement of adipokines in the pathophysiology of RA as suggested by in vitro ${ }^{1}$ and in vivo data $a^{2,3}$. The term "adipokines" was originally used to describe cytokine-like factors secreted by adipose tissue, but it turned out that many adipokines are also produced at other sites including the joints ${ }^{1,4,5}$, where they might have an influence on effector cells of RA or osteoarthritis (OA) pathophysiology.

As an adipokine, omentin was first found in omental adipose tissue from patients with Crohn disease ${ }^{6}$. Before this, it was identified as a secretory glycoprotein able to bind to galactofuranosyl residue on various microorganisms, suggesting a function in immune recognition of certain pathogens. It has also been described as a lactoferrin-binding protein with lactoferrin being a protein with multiple immunological functions. Omentin is highly abundant in human plasma ${ }^{6}$. It displayed antiinflammatory and antiatherogenic properties in obese patients ${ }^{7}$ and is negatively associated with inflammatory bowel disease (IBD) $)^{6,8}$ and metabolic syndrome ${ }^{9}$. Omentin levels in the synovial fluid (SF) of patients with RA were lower than in patients with $\mathrm{OA}^{5}$, while omentin serum levels were elevated in children with juvenile idiopathic arthritis compared with healthy controls and positively associated with the presence and number of active joints ${ }^{10}$.

Based on these previous findings, we decided to examine omentin expression in the synovium of patients with RA and OA. Synovial tissue samples obtained from patients with RA $(\mathrm{n}=12)$ and OA $(\mathrm{n}=10)$ undergoing joint surgery were analyzed immunohistochemically with a specific antiomentin antibody to determine the synovial omentin expression pattern. Immunohistochemical staining revealed omentin expression in RA and OA synovium, especially within the lining layer and vessel walls (Figure 1), and the same expression pattern for RA (Figure 1A and Figure 1B) and OA synovium (Figure 1C and Figure 1D). This similar expression pattern may also appear because synovial biopsies were from patients with late- or endstage RA or OA.

The presence of omentin in $\mathrm{SF}^{5}$ and synovial tissue led us to the hypothesis that this adipokine may also be involved in the pathogenesis of RA. Therefore, we analyzed the effects of omentin on the following key cells of RA pathophysiology: human synovial fibroblasts $(n=7)$, macrovascular endothelial cells $(E C)$ from varicose veins $(n=2)$, microvascular human umbilical artery EC $(\mathrm{n}=1)$, lymphocytes and monocytes from human blood $(\mathrm{n}=1)$, as well as chondrocytes from human cartilage $(\mathrm{n}=1)$. Because it had previously been shown that within the joint adipokines are particularly expressed by rheumatoid arthritis synovial fibroblasts (RASF) ${ }^{1}$, which can invade and destroy articular cartilage and subchondral bone, we focused our study on this cell type and started our analyses with RASF. All
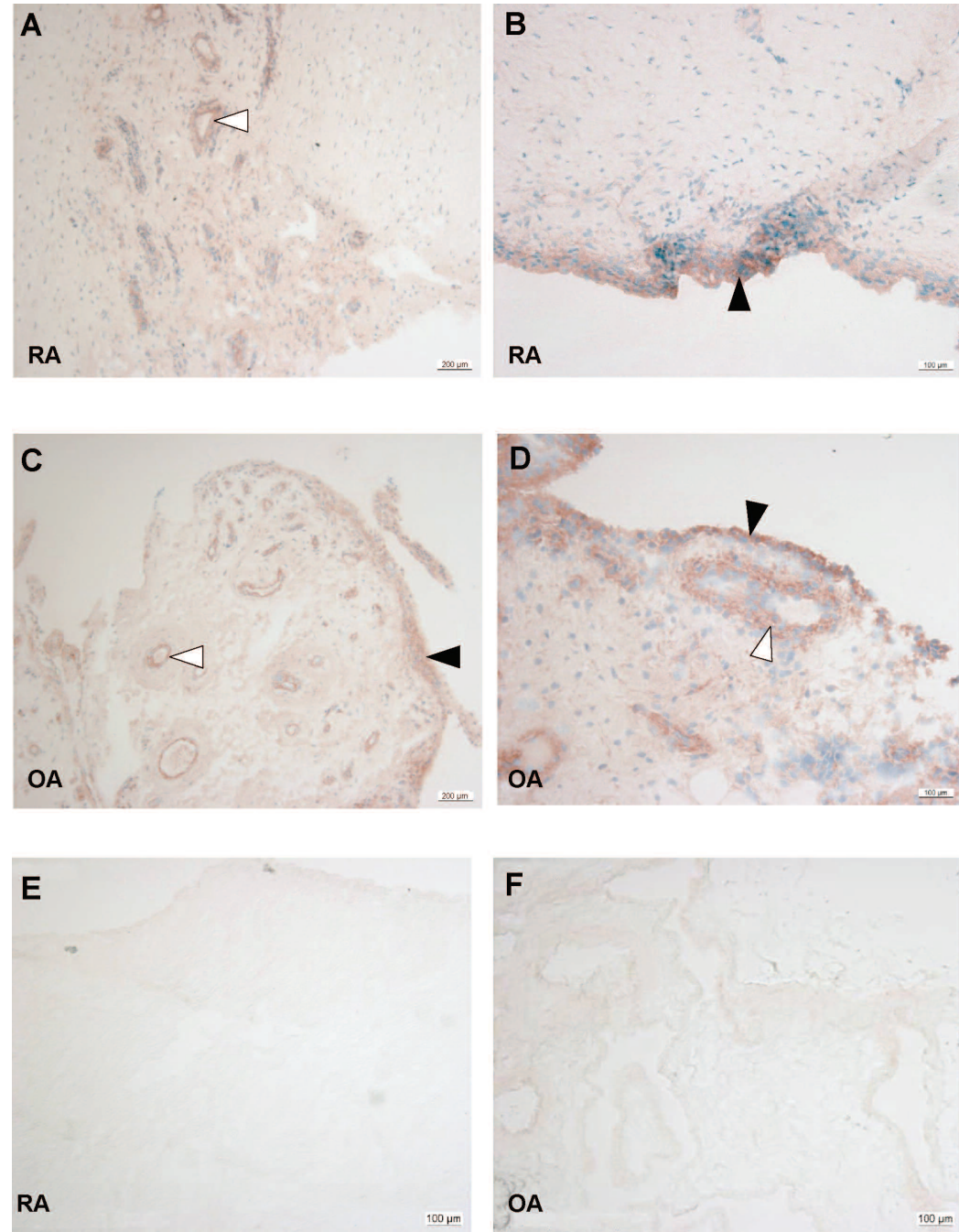

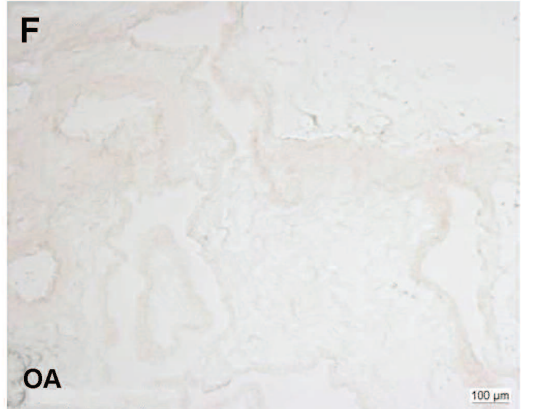

Figure 1. Expression of omentin in synovial tissue of patients with RA or OA. Immunostaining of omentin in synovial tissue was done with nuclear counterstaining (hematoxylin). Nuclei are stained in blue, and omentin is stained in red. A primary antibody isotype control is shown for RA and OA synovial tissue in $(\mathrm{E})$ and $(\mathrm{F})$. Expression of omentin in synovial tissue was most prominent within the synovial lining layer (black arrowheads) and perivascular (white arrowheads). RA synovial tissue (A and $\mathrm{B}$ ) showed the same expression pattern of omentin as OA synovial tissue (C and D). RA: rheumatoid arthritis; OA: osteoarthritis. 
cell types used were stimulated in vitro with $500 \mathrm{ng} / \mathrm{ml}$ omentin (BioVendor) for $15 \mathrm{~h}$. We first screened for potential changes in gene expressions induced by omentin in RASF using Affymetrix microarrays $(n=1$; human genome U133A oligonucleotide probe microarrays, Affymetrix) and antibody arrays ( $\mathrm{n}=1$; Human Chemokine Antibody Array I kit, custom human cytokine antibody array, RayBiotech). The array analysis resulted in a small number of genes or proteins showing limited up/downregulation by omentin in RASF (maximum upregulation: 13.1-fold, maximum downregulation: -13.9-fold; Table 1). However, the differential expression could not be confirmed for the genes selected for verification at mRNA level by real-time PCR or at protein level by ELISA (Table 1). For the other cell types, appropriate variables were analyzed by real-time PCR or ELISA; interleukin (IL)-6 was quantified for all cell types. Additionally, the following factors were determined in selected cell types: IL-8 for lymphocytes and monocytes; tumor necrosis factor- $\alpha$ only for lymphocytes; vascular endothelial growth factor and vascular cell adhesion molecule 1 for EC; and aggrecan, cartilage oligomeric matrix protein, and matrix metalloprotease 3 for chondrocytes
Interestingly, none of these factors were changed by omentin stimulation. Of note, the small sample sizes, which were not extended because of the negative characteristic of the results, may be a limitation of our study.

Although omentin is expressed in RA and OA synovium and present in $\mathrm{SF}$, it has no or limited effects on central effector cells of RA pathophysiology, in contrast to the effects observed with other adipokines ${ }^{1,4}$. Our data suggest that the potentially antiinflammatory effect of omentin in diseases such as obesity ${ }^{7}$ and IBD $^{8}$ does not occur in RA and OA, at least regarding the evaluated cell types.

KLAUS W. FROMMER, PhD, Department of Internal Medicine and Rheumatology, University of Giessen, Kerckhoff Clinic, Bad Nauheim, Germany; MASSIMILANO VASILE, MD, Department of Internal Medicine and Rheumatology, University of Giessen, Kerckhoff Clinic, Bad Nauheim, Germany, and Department of Medical Specialties, Division of Rheumatology, Sapienza University of Rome, Rome, Italy; ULF MÜLLER-LADNER, MD, Professor, University of Giessen, Department of

Table 1. Results of mRNA/protein expression analysis of omentin-stimulated RASF. RASF stimulated with $500 \mathrm{ng} / \mathrm{ml}$ omentin for $15 \mathrm{~h}$ and untreated controls were analyzed by Affymetrix (AFFX) oligonucleotide microarrays (GeneChip HG U133A) and protein arrays (RayBio "Custom Human Cytokine Antibody Array" and "Human Chemokine Antibody Array I"). The table shows a selection of functionally classified genes that according to the arrays were induced or repressed by omentin with fold changes of $\geq 2$ (induction) or $\leq-2$ (repression), respectively. For verification, selected genes/proteins were quantified by real-time PCR and ELISA.

\begin{tabular}{|c|c|c|c|c|c|}
\hline Gene/Protein & Symbol & $\begin{array}{c}\text { Fold Change } \\
\text { AFFX }\end{array}$ & $\begin{array}{l}\text { Fold Change } \\
\text { Real-time PCR }\end{array}$ & $\begin{array}{l}\text { Fold Change } \\
\text { Protein Arrays }\end{array}$ & $\begin{array}{l}\text { Fold Change } \\
\text { ELISA }\end{array}$ \\
\hline \multicolumn{6}{|l|}{ Chemokines } \\
\hline Chemokine (C-C motif) ligand 3 (CCL3) & MIP-1a & -7.6 & - & 2.7 & n.d. \\
\hline Chemokine (C-C motif) ligand 7 (CCL7) & MCP-3 & 8.5 & - & 4.6 & n.d. \\
\hline Chemokine (C-C motif) ligand 8 (CCL8) & MCP-2 & $\approx$ & - & 5.2 & - \\
\hline Chemokine (C-C motif) ligand 18 (CCL18) & PARC & $\approx$ & - & 4.3 & - \\
\hline Chemokine (C-C motif) ligand 20 (CCL20) & CCK1 & $\approx$ & - & 5.0 & - \\
\hline Chemokine (C-X-C motif) ligand 9 (CXCL9) & MIG & $\approx$ & - & 5.5 & n.d. \\
\hline Chemokine (C-X-C motif) ligand 10 (CXCL10) & IP-10 & -3.9 & n.d. & $\approx$ & - \\
\hline Chemokine (C-X-C motif) ligand 11 (CXCL11) & I-TAC & -5.5 & n.d. & $\approx$ & - \\
\hline \multicolumn{6}{|l|}{ Cytokines } \\
\hline Interleukin 3 & IL-3 & 4.5 & - & $\approx$ & - \\
\hline Interleukin 11 & IL-11 & 5.6 & - & $\varnothing$ & - \\
\hline Interleukin $17 \mathrm{C}$ & IL-17C & 3.7 & - & $\varnothing$ & - \\
\hline Tumor necrosis factor- $\alpha$ & TNF- $\alpha$ & $\approx$ & - & 4.7 & n.d. \\
\hline \multicolumn{6}{|l|}{ Miscellaneous inflammatory molecules } \\
\hline Prostaglandin endoperoxide synthase 1 & PTGS1 & 4.8 & - & $\varnothing$ & - \\
\hline \multicolumn{6}{|l|}{ Pre-B cell growth and B cell activation } \\
\hline Bone marrow stromal cell antigen 2 & BST2 & 3.0 & $\approx$ & $\varnothing$ & - \\
\hline \multicolumn{6}{|l|}{ Receptors } \\
\hline Chemokine (C-C) motif receptor 9 & CCR9 & -6.1 & - & $\varnothing$ & - \\
\hline Fibroblast growth factor receptor 3 & FGFR3 & 3.7 & - & $\varnothing$ & - \\
\hline \multicolumn{6}{|l|}{ Proteinases and peptidases } \\
\hline Matrix metallopeptidase 9 (gelatinase B) & MMP9 & 3.2 & - & 3.0 & - \\
\hline Matrix metallopeptidase 10 (stromelysin 2) & MMP10 & 3.2 & - & 2.5 & - \\
\hline Matrix metallopeptidase 14 & MMP14 & -4.0 & - & $\varnothing$ & - \\
\hline \multicolumn{6}{|l|}{ ECM and cell surface molecules } \\
\hline Collagen, type XXIV, alpha 1 & COL24A1 & -13.9 & - & $\varnothing$ & - \\
\hline Collagen, type VII, alpha 1 & COL7A1 & -4.8 & - & $\varnothing$ & - \\
\hline Glypican-3 & GPC3 & 13.1 & - & $\varnothing$ & - \\
\hline \multicolumn{6}{|l|}{ Bone and cartilage metabolism } \\
\hline Bone morphogenetic protein $8 \mathrm{~b}$ & BMP8B & 3.0 & - & $\varnothing$ & - \\
\hline \multicolumn{6}{|l|}{ Growth factors } \\
\hline Endothelial cell growth factor 1 (platelet-derived) & ECGF1 & 5.0 & - & $\varnothing$ & - \\
\hline Insulin-like growth factor 1 (somatomedin C) & IGF-1 & 2.0 & - & 10.8 & n.d. \\
\hline Vascular endothelial growth factor & VEGF & $\approx$ & - & 3.6 & - \\
\hline
\end{tabular}

RASF: rheumatoid arthritis synovial fibroblasts; ECM: extracellular matrix; $\approx$ : no change (i.e., less than \pm 2 -fold change); Ø: not present on array; n.d.: not detectable.

Personal non-commercial use only. The Journal of Rheumatology Copyright @ 2017 . All rights reserved. 
Rheumatology and Clinical Immunology, Kerckhoff Clinic, Bad Nauheim, Germany; ELENA NEUMANN, PhD, Department of Internal Medicine and Rheumatology, University of Giessen, Kerckhoff Clinic, Bad Nauheim,

Germany. Supported by the German Society for Rheumatology (Deutsche Gesellschaft für Rheumatologie). Address correspondence to Dr. K.W.

Frommer, Department of Internal Medicine and Rheumatology, University of Giessen, Kerckhoff Clinic, Benekestrasse 2-8, D-61231 Bad Nauheim,

Germany.E-mail: k.frommer@kerckhoff-klinik.de

\section{REFERENCES}

1. Neumann E, Frommer KW, Vasile M, Müller-Ladner U. Adipocytokines as driving forces in rheumatoid arthritis and related inflammatory diseases? Arthritis Rheum 2011;63:1159-69.

2. Rho YH, Solus J, Sokka T, Oeser A, Chung CP, Gebretsadik T, et al. Adipocytokines are associated with radiographic joint damage in rheumatoid arthritis. Arthritis Rheum 2009;60:1906-14.

3. Ebina K, Fukuhara A, Ando W, Hirao M, Koga T, Oshima K, et al. Serum adiponectin concentrations correlate with severity of rheumatoid arthritis evaluated by extent of joint destruction. Clin Rheumatol 2009;28:445-51.

4. Eisinger K, Bauer S, Schäffler A, Walter R, Neumann E, Buechler $\mathrm{C}$, et al. Chemerin induces CCL2 and TLR4 in synovial fibroblasts of patients with rheumatoid arthritis and osteoarthritis. Exp Mol Pathol 2012;92:90-6.
5. Senolt L, Polanská M, Filková M, Cerezo LA, Pavelka K, Gay S, et al. Vaspin and omentin: new adipokines differentially regulated at the site of inflammation in rheumatoid arthritis. Ann Rheum Dis 2009;69:1410-1.

6. Schäffler A, Neumeier M, Herfarth H, Fürst A, Schölmerich J, Büchler C. Genomic structure of human omentin, a new adipocytokine expressed in omental adipose tissue. Biochim Biophys Acta 2005;1732:96-102.

7. Zhou JY, Chan L, Zhou SW. Omentin: linking metabolic syndrome and cardiovascular disease. Curr Vasc Pharmacol 2014;12:136-43.

8. Yin J, Hou P, Wu Z, Nie Y. Decreased levels of serum omentin-1 in patients with inflammatory bowel disease. Med Sci Monit 2015;21:118-22.

9. Jaikanth C, Gurumurthy P, Cherian KM, Indhumathi T. Emergence of omentin as a pleiotropic adipocytokine. Exp Clin Endocrinol Diabetes 2013;121:377-83.

10. Cantarini L, Simonini G, Fioravanti A, Generoso M, Bacarelli MR, Dini E, et al. Circulating levels of the adipokines vaspin and omentin in patients with juvenile idiopathic arthritis, and relation to disease activity. Clin Exp Rheumatol 2011;29:1044-8.

J Rheumatol 2017;44:4; doi:10.3899/jrheum.161267 